\title{
Equivalence and axiomatization of solutions for cooperative games with circular communication structure
}

\author{
Ozer Selcuk* Takamasa Suzuki ${ }^{\dagger}$ Dolf Talman ${ }^{\ddagger}$
}

July 1, 2015

${ }^{*}$ O. Selcuk, CentER, Department of Econometrics \& Operations Research, Tilburg University, P.O. Box 90153, 5000 LE Tilburg, The Netherlands, e-mail: o.selcuk@tilburguniversity.edu.

${ }_{\dagger}^{\dagger}$ T. Suzuki, CentER, Department of Econometrics \& Operations Research, Tilburg University, P.O. Box 90153, 5000 LE Tilburg, The Netherlands, e-mail: t.suzuki@tilburguniversity.edu.

¥A.J.J. Talman, CentER, Department of Econometrics \& Operations Research, Tilburg University, P.O. Box 90153, 5000 LE Tilburg, The Netherlands, e-mail: talman@tilburguniversity.edu. 


\begin{abstract}
We study cooperative games with transferable utility and limited cooperation possibilities. The focus is on communication structures where the set of players forms a circle, so that the possibilities of cooperation are represented by the connected sets of nodes of an undirected circular graph. Single-valued solutions are considered which are the average of specific marginal vectors. A marginal vector is deduced from a permutation on the player set and assigns as payoff to a player his marginal contribution when he joins his predecessors in the permutation. We compare the collection of all marginal vectors that are deduced from the permutations in which every player is connected to his immediate predecessor with the one deduced from the permutations in which every player is connected to at least one of his predecessors. The average of the first collection yields the average tree solution and the average of the second one is the Shapley value for augmenting systems. Although the two collections of marginal vectors are different and the second collection contains the first one, it turns out that both solutions coincide on the class of circular graph games. Further, an axiomatization of the solution is given using efficiency, linearity, some restricted dummy property, and some kind of symmetry.
\end{abstract}

Keywords: Cooperative game, transferable utility, graph structure, marginal vectors, Shapley value, average tree solution

AMS subject classification: 90B18, 91A12, 91A43.

JEL code: C71. 


\section{Introduction}

A cooperative game with transferable utility, or TU-game, consists of a finite set of players and a characteristic function that assigns a worth to any subset of players. Players within such a coalition can freely distribute the worth of the coalition as payoff among themselves. The problem of a TU-game is how much payoff each player must receive. One of the most well-known single-valued solutions is the Shapley value ([7]) being the average of all marginal vectors of the game. A marginal vector is a payoff vector in which for some permutation on the player set each player receives as payoff his marginal contribution when he joins his predecessors in the permutation. The Shapley value is uniquely characterized by efficiency, the dummy property, linearity, and symmetry.

In many economic situations there exist restrictions which prevent some coalitions from cooperating. Myerson [6] introduces games with communication structure. Graph games arise when the restriction is represented by a graph in which the nodes of the graph represent the players and a link between two players indicates that they can communicate with each other. Only connected subsets of agents, called networks, are assumed to be able to form a coalition and attain their worth.

The average tree solution, introduced in [3] on the class of cycle-free graph games, is the average of the marginal vectors deduced from all spanning trees on the graph. A characterization using unanimity games on this class of graph games is given in [5]. In [4] the average tree solution is generalized to the class of arbitrary graph games by taking the average of the marginal vectors deduced from a specific subclass of spanning trees. On the class of non-cycle-free graph games characterizations using standard axioms or unanimity games are not known in the literature.

In this paper we consider the class of circle graph games where the underlying graph is assumed to be circular. Players could be firms or cities situated along a lake, around a mountain, or on a circular pipeline, where players can only be connected to their direct neighbors, one located on each side. In this setting a set of players is assumed only to be able to cooperate if they form a segment of the circle. We consider two different natural collections of permutations and take as solution concept the average of the deduced marginal vectors. The set of marginal vectors at which players are only able to contribute if they are connected to their immediate predecessor in the corresponding permutation appears to be the set of marginal vectors whose average is the average tree solution on the class of circle graph games, while the other collection at which players are only able to contribute if they are connected to one of their predecessors underlies the Shapley value introduced in [1] on the class of games on augmenting systems, which includes the class of circle graph games. Although the second set of marginal vectors contains the first set as a proper subset we show that the two solutions give the same payoff vector on the class of circle graph games. We further give for this class an axiomatization of the solution using standard axioms. The solution doesn't satisfy symmetry, but we show that it is fully characterized by efficiency, linearity, a restricted form of the dummy property, and some weak form of symmetry.

This paper is organized as follows. In Section 2 the class of circle graph games is introduced. 
In Section 3 several solutions are discussed and it is shown that they coincide on this class of games. In Section 4 an axiomatic characterization is given.

\section{Circle graph games}

Consider a finite set $N=\{1, \ldots, n\}$ of $n, n \geq 3$, players or agents located on a circle. Without loss of generality let $L=\{\{i, i+1\} \mid i=1, \ldots, n\}$ denote the set of links between the players, where $i+1=1$ when $i=n$. The pair $(N, L)$ is an undirected circle graph with the player set $N$ as the set of nodes and the link set $L$ as the set of edges. A coalition $S \in 2^{N}$ is connected, or a network, if for any $i, j \in S$ there is a sequence of different nodes $\left(i_{1}, i_{2}, \ldots, i_{k}\right)$ in $S$ such that $i_{1}=i, i_{k}=j$ and $\left\{i_{h}, i_{h+1}\right\} \in L$ for $h=1, \ldots, k-1$. The collection of networks in $(N, L)$ is denoted $C^{L}(N)$.

A permutation on the player set $N$ is a bijection on $N$ and represents an order in which the players can join each other to form the grand coalition $N$ of all players. $\Pi(N)$ denotes the set of all permutations on $N$. A permutation in $\Pi(N)$ is admissible if every player is connected to its immediate predecessor in the permutation. Let $\Pi^{a}(N)$ denote the set of admissible permutations on $N$, then

$$
\Pi^{a}(N)=\{\sigma \in \Pi(N) \mid\{\sigma(i), \sigma(i+1)\} \in L, i=1, \ldots, n-1\} .
$$

For every $i \in N$ there are two admissible permutations $\sigma$ with $\sigma(1)=i$, denoted by $\sigma_{1}^{i}=$ $(i, i+1, \ldots, n, 1, \ldots, i-1)$ and $\sigma_{2}^{i}=(i, i-1, \ldots, 1, n, \ldots, i+1)$, and so $\left|\Pi^{a}(N)\right|=2 n$ and $\Pi^{a}(N)=\left\{\sigma_{1}^{i} \mid i \in N\right\} \cup\left\{\sigma_{2}^{i} \mid i \in N\right\}$.

A permutation in $\Pi(N)$ is compatible if every player is connected to at least one of its predecessors, not necessarily the immediate predecessor in the permutation. Let $\Pi^{c}(N)$ denote the set of compatible permutations on $N$, then

$$
\Pi^{c}(N)=\left\{\sigma \in \Pi(N) \mid\{\sigma(1), \ldots, \sigma(k)\} \in C^{L}(N), k=1, \ldots, n-1\right\} .
$$

For every $i \in N$ there are two choices of $\sigma(2)$ for being compatible with $\sigma(1)=i$, namely $i-1$ and $i+1$, where $i-1=n$ if $i=1$ and $i+1=1$ if $i=n$. In general, for $k=2, \ldots, n-1$, there are two choices of $\sigma(k)$ for being compatible with $(\sigma(1), \sigma(2), \ldots, \sigma(k-1))$. Since $\sigma(n)$ is uniquely determined, this leads to $\left|\Pi^{c}(N)\right|=2^{n-2} n$. Notice that $\Pi^{a}(N) \subset \Pi^{c}(N)$.

A characteristic function $v: 2^{N} \rightarrow \mathbb{R}$, with $v(\emptyset)=0$, defines the worth of a coalition $S \in 2^{N}$. Following Myerson [6], only networks in $(N, L)$ are able to distribute freely their worth as payoff among its members. The triple $(N, v, L)$ is called a circle graph game and the class of circle graph games is denoted by $\mathcal{G}^{c}$. The problem of a circle graph game is how much payoff each player must receive. Given a circle graph game $(N, v, L) \in \mathcal{G}^{c}$, to any permutation $\sigma \in \Pi(N)$ a marginal (contribution) vector $m^{\sigma}(N, v, L)$ corresponds, assigning to agent $\sigma(k), k=1, \ldots, n$, as payoff

$$
m_{\sigma(k)}^{\sigma}(N, v, L)=v(\{\sigma(1), \ldots, \sigma(k)\})-v(\{\sigma(1), \ldots, \sigma(k-1)\}) .
$$




\section{Equivalent solutions}

The average tree solution, introduced in [3] on the class of cycle-free graph games and generalized in [4] to the class of arbitrary graph games, considers for circle graph games all $n$-tuples $B=$ $\left(B_{1}, \ldots, B_{n}\right)$ of networks in $(N, L)$ satisfying for every $i \in N$ that $i \in B_{i}$ and there is a unique $j \in N$ such that $\{i, j\} \in L$ and $B_{j}=B_{i} \backslash\{i\}$. Let $\mathcal{B}^{L}$ denote the collection of such admissible $n$-tuples of networks in $(N, L)$. Given a circle graph game $(N, v, L) \in \mathcal{G}^{c}$, to any $B \in \mathcal{B}^{L}$ a marginal vector $m^{B}(N, v, L)$ corresponds with payoff

$$
m_{i}^{B}(N, v, L)=v\left(B_{i}\right)-v\left(B_{i} \backslash\{i\}\right)
$$

for agent $i \in N$. The average tree solution, $A T$, assigns on the class of circle graph games to any game $(N, v, L) \in \mathcal{G}^{c}$ the average of the marginal vectors corresponding to all admissible $n$-tuples of networks in $(N, L)$, i.e.,

$$
A T(N, v, L)=\frac{1}{\left|\mathcal{B}^{L}\right|} \sum_{B \in \mathcal{B}^{L}} m^{B}(N, v, L) .
$$

First we show that on the class of circle graph games the average tree solution is the average of the marginal vectors induced by all admissible permutations.

Theorem 3.1 For any circle graph game $(N, v, L) \in \mathcal{G}^{c}$ it holds that

$$
A T(N, v, L)=\frac{1}{\left|\Pi^{a}(N)\right|} \sum_{\sigma \in \Pi^{a}(N)} m^{\sigma}(N, v, L) .
$$

Proof. Take any $\sigma \in \Pi^{a}(N)$ and suppose $\sigma=\sigma_{1}^{i}$ for some $i \in N$. Let $B_{k}=\{i, \ldots, k\}$ for $k=i, \ldots, n$ and $B_{k}=\{i, \ldots, n, 1, \ldots, k\}$ for $k=1, \ldots, i-1$. Then $B=\left(B_{1}, \ldots, B_{n}\right) \in \mathcal{B}^{L}$ and $m^{B}(N, v, L)=m^{\sigma_{1}^{i}}(N, v, L)$. Similarly, when $\sigma=\sigma_{2}^{i}$ for some $i \in N$, let $B_{k}=\{k, \ldots, i\}$ for $k=1, \ldots, i$ and $B_{k}=\{k, \ldots, n, 1, \ldots, i\}$ for $k=i+1, \ldots, n$. Then again $B=\left(B_{1}, \ldots, B_{n}\right) \in \mathcal{B}^{L}$ and $m^{B}(N, v, L)=m^{\sigma_{2}^{i}}(N, v, L)$.

Conversely, take any $B=\left(B_{1}, \ldots, B_{n}\right) \in \mathcal{B}^{L}$. There exists unique $i \in N$ such that $B_{i}=N$. Then either $B_{i} \backslash\{i\}=B_{i+1}\left(B_{1}\right.$ if $\left.i=n\right)$ or $B_{i-1}\left(B_{n}\right.$ if $\left.i=1\right)$. Suppose $B_{i} \backslash\{i\}=B_{i+1}$. Then, when $i<n, i+2(1$ if $i=n-1)$ is the only element of $B_{i+1} \backslash\{i+1\}$ that is linked in $L$ to $i+1$ and so $B_{i+1} \backslash\{i+1\}=B_{i+2}$, and, when $i=n, 2$ is the only element of $B_{1} \backslash\{1\}$ that is linked to 1 . In general, for $k=i+1, \ldots, n, 1, \ldots, i$ only element $k+1$ in $B_{k} \backslash\{k\}$ is linked to $k$ and so $B_{k} \backslash\{k\}=B_{k+1}$. From this it follows that $m^{B}(N, v, L)=m_{2}^{\sigma_{2}^{i-1}}(N, v, L)$. Similarly, if $B_{i} \backslash\{i\}=B_{i-1}$, it holds that $m^{B}(N, v, L)=m^{\sigma_{1}^{i+1}}(N, v, L)$. Thus every admissible $n$-tuple of networks in $(N, L)$ corresponds to a unique admissible permutation in $\Pi^{a}(N)$.

As solution concept we may also consider the average of the marginal vectors corresponding to all compatible permutations. On the class of circle graph games, this is equivalent to the Shapley value introduced in Bilbao and Ordóñez [1] on the class of games with augmenting systems, which contains the class of circle graph games. Given a circle graph game $(N, v, L) \in \mathcal{G}^{c}$, the Shapley value in [1] considers the collection of maximal chains of $(N, L)$. A maximal chain is 
a collection of networks ordered with respect to set inclusion that is not contained in any larger chain. It is easily seen that the length of each maximal chain is $n$ and that each maximal chain corresponds one-to-one to a compatible permutation.

For the average tree solution it is assumed that a player is able to contribute only if he is connected to his immediate predecessor and for the Shapley value in [1] if he is connected to at least one predecessor. Although the number of the marginal vectors differ and generically all compatible marginal vectors are different and contain the admissible marginal vectors as a proper subset, the two averages yield the same payoff vector on the class of circle graph games. ${ }^{1}$

Theorem 3.2 On the class of circle graph games the average tree solution and the Shapley value in [1] coincide.

Proof. For any circle graph game $(N, v, L) \in \mathcal{G}^{c}$ it has to be shown that

$$
\frac{1}{\left|\Pi^{a}(N)\right|} \sum_{\sigma \in \Pi^{a}(N)} m^{\sigma}(N, v, L)=\frac{1}{\left|\Pi^{c}(N)\right|} \sum_{\sigma \in \Pi^{c}(N)} m^{\sigma}(N, v, L) .
$$

Take any $S \in C^{L}(N)$ and $i \notin S$ satisfying $S \cup\{i\} \in C^{L}(N)$. Let $\Pi_{S, i}^{a}\left(\Pi_{S, i}^{c}\right)$ denote the subsets of admissible (compatible) permutations $\sigma$ satisfying $m_{i}^{\sigma}(N, v, L)=v(S \cup\{i\})-v(S)$. It is to show that $\frac{\left|\Pi_{S, i}^{a}\right|}{\left|\Pi^{a}(N)\right|}=\frac{\left|\Pi_{S, i}^{c}\right|}{\left|\Pi^{c}(N)\right|}$. If $S=\emptyset$, then $\Pi_{S, i}^{a}=\left\{\sigma_{1}^{i}, \sigma_{2}^{i}\right\}$ and $\Pi_{S, i}^{c}$ consists of $2^{n-2}$ compatible permutations $\sigma$ with $\sigma(1)=i$, and therefore $\frac{\left|\Pi_{S, i}^{a}\right|}{\left|\Pi^{a}(N)\right|}=\frac{1}{n}=\frac{\left|\Pi_{S, i}^{c}\right|}{\left|\Pi^{c}(N)\right|}$. If $S=N \backslash\{i\}$, then $\Pi_{S, i}^{a}=\left\{\sigma_{1}^{i+1}, \sigma_{2}^{i-1}\right\}$ and $\Pi_{S, i}^{c}$ consists of $2^{n-2}$ compatible permutations $\sigma$ with $\sigma(n)=i$, and therefore $\frac{\left|\Pi_{S, i}^{a}\right|}{\left|\Pi^{a}(N)\right|}=\frac{1}{n}=\frac{\left|\Pi_{S, i}^{c}\right|}{\left|\Pi^{c}(N)\right|}$. Otherwise, $\left|\Pi_{S, i}^{a}\right|=1$ and $\left|\Pi_{S, i}^{c}\right|=2^{|S|-1} \cdot 2^{n-|S|-2}=2^{n-3}$, where the first term of the product is the number of ways to fill the first $|S|$ positions and the second term is the number of ways to fill the last $n-|S|-1$ positions. Therefore it holds that $\frac{\left|\Pi_{S, i}^{a}(N)\right|}{\left|\Pi^{a}(N)\right|}=\frac{1}{2 n}=\frac{\left|\Pi_{S, i}^{c}\right|}{\left|\Pi^{c}(N)\right|}$ for any such $S$, which completes the proof.

\section{Axiomatic characterization}

In this section we axiomatize the average tree solution and therefore also the Shapley value in [1] on the class of circle graph games. The first two axioms are standard.

Definition 4.1 A solution $\xi: \mathcal{G}^{c} \rightarrow \mathbb{R}^{n}$ satisfies efficiency if for any $(N, v, L) \in \mathcal{G}^{c}$ it holds that $\sum_{i \in N} \xi_{i}(N, v, L)=v(N)$.

Definition 4.2 A solution $\xi: \mathcal{G}^{c} \rightarrow \mathbb{R}^{n}$ satisfies linearity if for any $(N, v, L),(N, w, L) \in \mathcal{G}^{c}$ and $a, b \in \mathbb{R}$ it holds that $\xi(N, a v+b w, L)=a \xi(N, v, L)+b \xi(N, w, L)$.

A player $i \in N$ is a restricted dummy player in a circle graph game $(N, v, L) \in \mathcal{G}^{c}$ if this player never contributes whenever he joins a network to form a new network, i.e., $v(S \cup\{i\})-$ $v(S)=0$ for all $S \in C^{L}(N)$ satisfying $i \notin S$ and $S \cup\{i\} \in C^{L}(N)$. This player must get zero payoff.

\footnotetext{
${ }^{1}$ For graph games in which the graph is complete both solutions are equal to the Shapley value of the game. For other graph games the average tree solution and the Shapley value in [1] will differ.
} 
Definition 4.3 A solution $\xi: \mathcal{G}^{c} \rightarrow \mathbb{R}^{n}$ satisfies the restricted dummy property if for any $(N, v, L) \in \mathcal{G}^{c}$ and restricted dummy player $i \in N$ in $(N, v, L)$ it holds that $\xi_{i}(N, v, L)=0$.

The next two axioms together are a weak form of symmetry.

Definition 4.4 A solution $\xi: \mathcal{G}^{c} \rightarrow \mathbb{R}^{n}$ satisfies symmetry among players if for any admissible permutation $\pi \in \Pi^{a}(N)$ it holds that $\xi_{i}\left(N, v^{\prime}, L\right)=\xi_{\pi(i)}(N, v, L)$, where $v^{\prime}(S)=v(\pi(S))$ for all $S \in C^{L}(N)$.

Symmetry among players means that if two circle graph games differ only by a shift or reversed shift of the players along the circle, then the solution also differs only by this shift.

Definition 4.5 A solution $\xi: \mathcal{G}^{c} \rightarrow \mathbb{R}^{n}$ satisfies symmetry between games if for any $v, v^{\prime} \in \mathcal{G}^{c}$ and $i \in N$ it holds that $\xi_{i}(N, v, L)=\xi_{i}\left(N, v^{\prime}, L\right)$ when $v(S)=v^{\prime}(S)$ and $v(S \cup\{i\})=v^{\prime}(S \cup\{i\})$ for all $S \in C^{L}(N)$ satisfying $i \notin S$ and $S \cup\{i\} \in C^{L}(N)$.

Symmetry between games implies that in two different circle graph games a player gets the same payoff if in both games the worth of any network to which this player is connected is the same and also the worth of such a network together with this player is the same.

To prove that on the class of circle graph games the axioms above uniquely define the average tree solution, we need unanimity games. For $T \in C^{L}(N)$, the unanimity game $u_{T}$ is given by $u_{T}(S)=1$ if $T \subseteq S$ and 0 otherwise. A player $j \in T$ is an end player of network $T$ if $T \backslash\{j\} \in$ $C^{L}(N)$. Let $E(T)$ denote the set of end players of network $T$. For the unanimity circle graph game $\left(N, u_{T}, L\right), T \in C^{L}(N)$, it holds that $A T_{j}\left(N, u_{T}, L\right)=1$ if $T=\{j\}, A T_{j}\left(N, u_{T}, L\right)=\frac{1}{n}$ if $j \in T \backslash E(T), A T_{j}\left(N, u_{T}, L\right)=(n+2-|T|) /(2 n)$ if $j \in E(T)$ and $|T|>1$, and $A T_{j}\left(N, u_{T}, L\right)=0$ if $j \notin T$.

Theorem 4.6 On the class of circle graph games the average tree solution is the unique solution satisfying efficiency, linearity, the restricted dummy property, symmetry among players, and symmetry between games.

Proof First, we show that the average tree solution satisfies all properties. Efficiency follows from the fact that all marginal vectors are efficient by construction. Since all admissible marginal vectors of a circle graph game are linear in the worths of the networks and the average tree solution is the average of these vectors, the average tree solution satisfies linearity. If a player is a restricted dummy player, this player has marginal contribution equal to zero at any admissible permutation and therefore the average is also zero. If players are shifted or reversely shifted along the circle the marginal vectors corresponding to admissible permutations shift accordingly and therefore also their average. Finally, if in two different games a player has the same marginal contribution to any network he is connected to, the average tree solution assigns to that player the same payoff in both games.

Second, let $\xi$ be a solution which satisfies all five axioms. The proof is first done for the class of unanimity circle graph games. Consider the unanimity game $u_{N}$. Take $\pi=\sigma_{1}^{k}$ for any 
$k \neq 1$. Then $\pi \in \Pi^{a}(N)$ and $u_{N}(\pi(S))=u_{N}(S)$ for all $S \in C^{L}(N)$. By symmetry among players it follows that $\xi_{1}\left(N, u_{N}, L\right)=\xi_{k}\left(N, u_{N}, L\right)$, which implies that in the game $u_{N}$ all players receive the same payoff. By efficiency, this yields

$$
\xi_{k}\left(N, u_{N}, L\right)=\frac{1}{n}=A T_{k}\left(N, u_{N}, L\right) \quad \forall k \in N .
$$

For the unanimity game $u_{T}$ with $T=\{i\}$ it follows from efficiency and the restricted dummy property that player $i$ receives 1 and all other players 0 as in the average tree solution. Now, take any $T \in C^{L}(N)$ with $1<|T|<n$. Then each $i \notin T$ is a restricted dummy player in $\left(N, u_{T}, L\right)$ and therefore this player receives zero payoff as in the average tree solution. Next, let $i \in T \backslash E(T)$, then for all $S \in C^{L}(N)$ such that $i \notin S$ and $S \cup\{i\} \in C^{L}(N)$ it holds that $u_{T}(S \cup\{i\})=u_{N}(S \cup\{i\})$. From symmetry between games it follows that

$$
\xi_{i}\left(N, u_{T}, L\right)=\xi_{i}\left(N, u_{N}, L\right)=\frac{1}{n}=A T_{i}\left(N, u_{T}, L\right) .
$$

Finally, let $i \in E(T)$. Because of symmetry among players and since $1<|T|<n$, we may assume that $i=1$ and $T=\{1, \ldots, j\}$ for some $1<j<n$. Let $\pi=\sigma_{2}^{j}$, then $\pi \in \Pi^{a}(N), \pi(1)=j$, and $\pi(T)=T$. Define the game $v^{\prime}$ by $v^{\prime}(S)=u_{T}(\pi(S))$ for all $S \in 2^{N}$. Because of symmetry among players and since $\pi(1)=j$, it holds that $\xi_{j}\left(N, v^{\prime}, L\right)=\xi_{1}\left(N, u_{T}, L\right)$. Moreover, $v^{\prime}(S \cup\{j\})=1=$ $u_{T}(S \cup\{j\})$ if $S \in C^{L}(N)$ and $S \supseteq T \backslash\{j\}$, and $v^{\prime}(S \cup\{j\})=0=u_{T}(S \cup\{j\})$ for all other $S \in C^{L}(N)$. From symmetry between games it then follows that $\xi_{j}\left(N, v^{\prime}, L\right)=\xi_{j}\left(N, u_{T}, L\right)$. Together this implies $\xi_{j}\left(N, u_{T}, L\right)=\xi_{1}\left(N, u_{T}, L\right)$, and so the two end players of $T$ receive the same payoff in the unanimity circle graph game $\left(N, u_{T}, L\right)$. From efficiency and the facts that all other players in $T$ receive payoff $\frac{1}{n}$ and all players outside $T$ receive payoff zero, the two end players 1 and $j$ of network $T$ receive both payoff equal to

$$
\xi_{1}\left(N, u_{T}, L\right)=\xi_{j}\left(N, u_{T}, L\right)=\frac{1}{2}\left(1-\frac{|T|-2}{n}\right)=\frac{n+2-|T|}{2 n},
$$

which is the same as both players receive at the average tree solution. Thus $\xi\left(N, u_{T}, L\right)=$ $A T\left(N, u_{T}, L\right)$ for any $T \in C^{L}(N)$. The proof is concluded since on the collection of networks any circle graph game can be expressed as a linear combination of unanimity circle graph games.

To show the independence of the five axioms, consider the linear solution $\xi(N, v, L)=$ $\sum_{T \in C^{L}(N)} c_{T} f\left(N, u_{T}, L\right)$ where $v=\sum_{T \in C^{L}(N)} c_{T} u_{T}$ and $f\left(N, u_{T}, L\right)$ allocates $\frac{1}{|T|}$ to players in $T$ and 0 to players not in $T$. It only fails symmetry between games because the non-end players of some proper network $T$ with $|T|>2$ receive different payoffs in the unanimity circle graph games $\left(N, u_{T}, L\right)$ and $\left(N, u_{N}, L\right)$. Next, consider the linear solution $\pi(N, v, L)=$ $\sum_{T \in C^{L}(N)} c_{T} f\left(N, u_{T}, L\right)$ where $v=\sum_{T \in C^{L}(N)} c_{T} u_{T}$ and $f\left(N, u_{T}, L\right)$ allocates 1 to the end player who has the smallest index and 0 to any other player. If $|T|>1$, symmetry among players fails to hold, while the other axioms hold. Next, let $D(v)$ be the set of restricted dummy players of a circle graph game $(N, v, L) \in \mathcal{G}^{c}$ and consider the solution that allocates to a circle graph game $(N, v, L) \in \mathcal{G}^{c}$ the payoff vector $\xi(N, v, L)$ as follows. If there is $i \in N$ for which there exists $T \in C^{L}(N)$ satisfying $v(S)=u_{T}(S)$ and $v(S \cup\{i\})=u_{T}(S \cup\{i\})$ for all $S \in C^{L}(N)$ such that 
$i \notin S$ and $S \cup\{i\} \in C^{L}(N)$, then for all such $i$ we take $\xi_{i}(N, v, L)=A T_{i}\left(N, u_{T}, L\right)$, each player in $D(v)$ receives payoff 0 , and all other players equally share the remaining payoff. If for all $i \in N$ such $T$ does not exist, then we take $\xi_{j}(N, v, L)=0$ if $j \in D(v)$ and $\xi_{j}(N, v, L)=v(N) /(n-|D(v)|)$ if $j \notin D(v)$. This solution satisfies all axioms except linearity. The equal sharing solution, where each agent receives $v(N) / n$, satisfies every axiom except the restricted dummy property. Finally, the solution where each agent receives zero payoff only fails efficiency.

\section{References}

[1] Bilbao, J.M. and M. Ordóñez (2009), Axiomatizations of the Shapley value for games on augmenting systems, European Journal of Operations Research, 196, 1008-1014.

[2] Borm, P., G. Owen, and S. Tijs (1992), On the position value for communication situations, SIAM Journal of Discrete Mathematics, 5, 305-320.

[3] Herings, P.J.J., G. van der Laan, and A.J.J. Talman (2008), The average tree solution for cycle-free graph games, Games and Economic Behavior, 62, 77-92.

[4] Herings, P.J.J., G. van der Laan, A.J.J. Talman, and Z. Yang (2010), The average tree solution for cooperative games with communication structure, Games and Economic Behavior, $68,626-632$.

[5] Mishra, D. and A.J.J. Talman (2010), A characterization of the average tree solution for tree games, International Journal of Game Theory, 39, 105-111.

[6] Myerson, R.B. (1977), Graphs and cooperation in games, Mathematics of Operations Research, 2, 225-229.

[7] Shapley, L. (1953), A value for $n$-person games, in H.W. Kuhn and A.W. Tucker (eds.), Contributions to the Theory of Games II, Princeton University Press, Princeton, pp. 307317. 